\title{
Inflation of the Endotracheal Tube Cuff in the Pharynx for Ventilation of Paralyzed Patients with Unanticipated Difficult Airway
}

\author{
Alfredo Panadero, M.D., Ph.D. \\ Pablo Monedero, M.D., Ph.D. [pmonedero@unav.es] \\ Isidro Olavide, M.D., Ph.D. \\ Ignacio Fernández-Liesa, M.D., Ph.D. \\ Jose Manuel Mendieta, M.D., Ph.D. \\ Antonio Macías, M.D. \\ Department of Anesthesia and Critical Care \\ Clínica Universitaria de Navarra \\ School of Medicine \\ University of Navarra \\ Pamplona, Spain
}

Airway management is essential to anesthesia practice. Despite several predictors of difficult intubation, patients with unexpectedly difficult airways are sometimes encountered, and an unforeseen grade III-IV laryngoscopy in a paralyzed patient is still a potentially dangerous situation. Several techniques and devices can be used in the face of an unplanned difficult intubation, ${ }^{1}$ such as the laryngeal mask airway, the Combitube, COPA, and light wand. However, there are situations in which these techniques and devices may not be available.

There is a simple technique that will allow ventilation of the paralyzed patient for several minutes until help or alternative methods arrive. The technique involves the placement of an endotracheal tube into the pharynx, with its tip just below the epiglottis, followed by inflation of the cuff with a large volume of air. Ventilation is then performed via the endotracheal tube.

To confirm the usefulness of this method, we conducted a clinical study in 50 patients with American Society of Anesthesiologists physical status I-II (27 men) undergoing general anesthesia. The hospital ethical committee approved the study, and informed consent was obtained from the participants. Exclusion criteria were age $<18$ yr or $>70$ $\mathrm{yr}$, last oral intake less than $8 \mathrm{~h}$, and hiatal hernia or gastroesophageal reflux. The patients all had normal airways on examination (Mallampati class I-II, normal neck extension, hyomental distance of $>6 \mathrm{~cm}$ ). After induction of anesthesia and neuromuscular blockade, a laryngoscope was inserted until only the tip of the epiglottis was observed. An endotracheal tube with a 7.5-8 mm internal diameter (Mallinckrodt) with Murphy eye was introduced, placing the tip under the epiglottis with the cuff of the tube remaining in full view. In this position, the cuff was inflated with 50-60 ml of air by an assistant. We connected the tube to the circuit, and the patients were manually ventilated at a rate of approximately 25 breaths per minute. We checked appropriate ventilation by noting the movement of the chest and the presence of expired $\mathrm{CO}_{2}$, and by auscultation of the lungs and epigastrium. The hyperinflated cuff produces an 
oropharyngeal seal. After $3 \mathrm{~min}$ of ventilation, the cuff was deflated, the tube was removed, and a new endotracheal tube was placed through the cords for subsequent ventilation. We were able to ventilate all patients without difficulty, although the endotracheal tube occasionally needed to be withdrawn slightly to correct an air leak. Oxygen saturation as measured by pulse oximetry $\left(\mathrm{Sp}_{\mathrm{O}}\right)$ was maintained at $>95 \%$ in all patients. No patients complained of sore throat in the postoperative period.

During the study period, two additional patients who were not involved in the study were discovered to have an unexpectedly difficult laryngoscopy after anesthetic induction. We used the described method successfully in both cases. We also passed a flexible fiberoptic laryngoscope through the tubes. After partially deflating the cuff, we saw the glottic opening, and the fiberoptic laryngoscope was introduced easily.

Endotracheal-tube cuff inflation in the oropharynx has been described previously as an aid to blind nasotracheal intubation with spontaneous ventilation, both in normal patients $^{2-3}$ and in those with altered anatomy. ${ }^{4}$ The currently described technique could be used in the setting of the unanticipated difficult airway, when other means of establishing a patent airway are not available.

\section{REFERENCES}

1. Practice guidelines for management of the difficult airway. A report by the American Society of Anesthesiologists Task Force on Management of the Difficult Airway. Anesthesiology 1993; 78:597-602.

2. Van Elstraete AC, Mamie JC, Mehdaoui H: Nasotracheal intubation in patients with immobilized cervical spine: A comparison of tracheal tube cuff inflation and fiberoptic bronchoscopy. Anesth Analg 1998; 87:400-2.

3. Gorback MS: Inflation of the endotracheal tube cuff as an aid to blind nasal endotracheal intubation. Anesth Analg 1987; 66: 916-7.

4. Casals-Caus P, Mayoral-Rojals V, Canales MA, Ruiz-Tamarit V, Casals-Castells A, Cochs-Cristia J: Inflation of the endotracheal tube cuff as an aid for blind nasotracheal intubation in patients with predicted difficult laryngoscopy. Rev Esp Anestesiol Reanim 1997; 44:302-4. 